\title{
Polarization of Instantons and Gravity
}

\author{
M. Yu. KuchievL \\ School of Physics, University of New South Wales, Sydney, 2052, Australia
}

(June 21, 2017)

Gravity can arise in a conventional non-Abelian gauge theory in which a specific phenomenon takes place. Suppose there is a condensation of polarized instantons and antiinstantons in the vacuum state. Then the excitations of the gauge field in the classical approximation are described through the variables of Riemann geometry satisfying the Einstein equations of general relativity. There are no dimensional coupling constants in the theory.

PACS: $04.60,12.25$

In this work I want to demonstrate that conventional gauge theory can provide the basis for gravity. The theory under consideration is the usual non-Abelian gauge field theory [1] dealing exclusively with particles of spin $0,1 / 2$ and 1 . There are no gravitons on the basic level. Space-time is fundamentally flat. There are no dimensional coupling constants in the theory.

Obviously there must appear something nontrivial in the usual gauge theory which yields the gravitational effect. This is a specific phenomenon in which instantons [2] play a crucial role. Generally speaking an instanton may be oriented arbitrarily. Let us assume that the vacuum state possesses a condensate of instantons with a specific property: the instantons belonging to this condensate have a preferred orientation. We shall call them "polarized" instantons. More precisely we suppose the existence of two condensates. One condensate consists of polarized instantons belonging to some $s u(2)$ sub-algebra of the gauge algebra. The other is a condensate of polarized antiinstantons belonging to some other $s u(2)$ subalgebra of the gauge algebra. We shall call this property the Instanton-Antiinstanton Polarization (IAP).

Suppose that there is a non-Abelian gauge theory in which there is IAP in the vacuum state. Then this theory is shown to describe the effects of gravity. Gravity arises due to a smooth variation of the orientation of the condensates of instantons and antiinstantons in space-time. The main quantities which describe gravity - the metric, Christoffel symbols, Riemann tensor - are functions of the gauge field. The Einstein equations of general relativity are shown to arise as a classical approximation for this gauge theory. These statements are valid on the classical level only. The basic quantum theory is the theory of the gauge field in flat space-time. The possibility of constructing a gauge theory with the IAP is not discussed in this paper, - it needs special consideration.

The mathematical resemblance between the variables in general relativity and in gauge theories is well known, see [3]. In [4] there was developed the approach which in some cases helps to clarify this resemblance. Certainly there are distinctions. The most important for our consideration are the following:

i. General relativity is invariant under the local Lorentz transformations. The Lorentz group is non-compact. In contrast to that the successful gauge theories are based upon a compact group of gauge transformations.

ii. The Lagrangian for the gravitational field in general relativity is linear with respect to the Riemann tensor (defined in accordance with [6])

$$
\mathcal{L}_{G R}=-\frac{1}{16 \pi k} \sqrt{-g} g^{\lambda \mu} g^{\rho \nu} R_{\lambda \rho \mu \nu}
$$

while the Lagrangian for the gauge field is quadratic with respect to $F_{\mu \nu}^{i j}$,

$$
\mathcal{L}_{Y M}=-\frac{1}{4 g^{2}} F_{\mu \nu}^{i j} F_{\mu \nu}^{i j}
$$

The obvious resemblance between the Riemann tensor and the strength of a gauge field does not manifest itself on the level of the Lagrangians. This distinction makes the classical equations of motion in the two theories to be quite different. In gravity theories with higher covariant derivatives, see [5], the Lagrangian is more sophisticated then in Eq.(1), but still the linear in $R_{\lambda \rho \mu \nu}$ term plays a role, preventing the gravity Lagrangian to be equal to the Yang-Mills Lagrangian.

iii. The basic quantity in general relativity is the metric, the Christoffel symbols $\Gamma_{\mu \nu}^{\lambda}$ being derived from it. On the other hand in gauge theories the vector potential $A_{\mu}^{i j}$ plays the major role. Again, the resemblance between the Christoffel symbols and the vector potential is not manifested in the dynamical properties of the two theories.

We will see that IAP is a remedy which sweeps away all these distinctions. The first question to be addressed is the gauge group. The local Lorentz group is characterized by six parameters. If a gauge theory is believed to describe the effects of gravity then the number of parameters of the gauge group is to be six (or more). The simplest compact group with six parameters is $\mathrm{SO}(4)$. Therefore let us consider the usual $\mathrm{SO}(4)$ gauge theory with the conventional Lagrangian which includes the term (2) describing the non-Abelian gauge field. Gauge theories may differ in the number of generations of the scalars and fermions, their masses and coupling constants which results in a variety of properties of the theories. In this work we assume that among these theories there exists a gauge theory with the necessary property - IAP.

The considered gauge algebra so(4) is the direct sum of two $s u(2), s o(4)=s u(2)+s u(2)$. We can choose the generators for one $s u(2)$ to be $(-1 / 2) \eta_{a i j}$ and the generators for the other to be $(-1 / 2) \bar{\eta}_{a i j}$, and refer to these algebras as $s u(2) \eta$ and $s u(2) \bar{\eta}$. Here $\eta_{a i j}, \bar{\eta}_{a i j}$ are the 't Hooft symbols, $a=1,2,3, i j=1, \cdots, 4$. The strength of the gauge field in this notation is

$$
F_{\mu \nu}^{i j}=-(1 / 2)\left(F_{\mu \nu}^{a} \eta_{a i j}+\bar{F}_{\mu \nu}^{a} \bar{\eta}_{a i j}\right)
$$

where $F_{\mu \nu}^{a}$ belongs to $s u(2) \eta$ and $\bar{F}_{\mu \nu}^{a}$ belongs to $s u(2) \bar{\eta}$.

We assume that there is a condensate of polarized instantons belonging to $s u(2) \bar{\eta}$ and a condensate of polarized antiinstantons belonging to $s u(2) \eta$. Now let us consider the interaction of the sufficiently weak and slowly 
varying gauge field with this polarized state. From now on the Euclidean formulation is used. The interaction of one instanton with a smoothly varying weak field was considered in Ref. [7], see also [8]. For the case of the $S U(2)$ gauge group the effective action describing this interaction is

$$
S_{i n s}=\left(2 \pi^{2} \rho^{2} / g^{2}\right) \bar{\eta}_{a \mu \nu} \bar{F}_{\mu \nu}^{b} \bar{C}_{a b}
$$

where $\rho$ is the radius of instanton, and $\bar{C}_{a b} \in S O(3)$ is a matrix describing the orientation of the instanton. The bar over $\bar{F}_{\mu \nu}^{b}$ reminds us that the instanton under consideration belongs to $s u(2) \bar{\eta}$. Eq.(4) remains valid for the antiinstanton as well if we substitute $\bar{\eta}_{a \mu \nu} \rightarrow \eta_{a \mu \nu}, \bar{F}_{\mu \nu}^{b} \rightarrow F_{\mu \nu}^{b}$, and $\bar{C}_{a b} \rightarrow C_{a b}$, where $C_{a b} \in S O(3)$ is a matrix describing the orientation of the antiinstanton.

Now let us apply this result to the vacuum with IAP. If we suppose the dilute gas approximation for the polarized instantons and antiinstantons to be valid then from Eq. (4) with the help of Eq.(3) we deduce that the interaction of the slowly varying weak field with this vacuum is described by the Lagrangian

$$
\mathcal{L}=-\left(\eta_{a \mu \nu} \eta_{b i j} M_{a b}+\bar{\eta}_{a \mu \nu} \bar{\eta}_{b i j} \bar{M}_{a b}\right) F_{\mu \nu}^{i j} .
$$

Here $M_{a b}, \bar{M}_{a b}$ are defined as

$$
\begin{aligned}
& M_{a b}=\pi^{2}\left\langle\left(1 / g^{2}\right) \rho^{2} n(\rho, C) C_{a b}\right\rangle, \\
& \bar{M}_{a b}=\pi^{2}\left\langle\left(1 / g^{2}\right) \rho^{2} \bar{n}(\rho, \bar{C}) \bar{C}_{a b}\right\rangle,
\end{aligned}
$$

where $n(\rho, C)$ is the concentration of the $s u(2) \bar{\eta}$ instantons with radius $\rho$ and orientation given by the matrix $C_{a b}$, and $\bar{n}(\rho, \bar{C})$ is the concentration of the $s u(2) \eta$ antiinstantons with orientation given by the matrix $\bar{C}_{a b}$. The brackets \langle\rangle in $([6),(7)$ describe the average over microscopic fluctuations of the gauge field. The existence of IAP means that there are condensates of polarized $s u(2) \bar{\eta}$ instantons and $s u(2) \eta$ antiinstantons which give the nonzero contribution to the right-hand sides of (6), (7)

$$
M_{a b}=(f / 4) C_{a b}^{c o n d}, \quad \bar{M}_{a b}=(\bar{f} / 4) \bar{C}_{a b}^{c o n d},
$$

where $C_{a b}^{c o n d} \in S O(3)$ is the matrix describing the orientation of the condensate of polarized instantons and $\bar{C}_{a b}^{\text {cond }} \in S O(3)$ is the matrix describing the orientation of the condensate of polarized antiinstantons. The constants $f, \bar{f}$ characterize the intensity of these condensates. We will suppose them to be equal, and hence $f=\bar{f}$.

It is useful to present the matrixes $C_{a b}^{c o n d}, \bar{C}_{a b}^{c o n d}$ with the help of a matrix $h^{i j} \in S O(4)$ which satisfies the equations

$$
h^{i k} h^{j l} \eta_{a k l}=C_{a b}^{c o n d} \eta_{b i j}, \quad h^{i k} h^{j l} \bar{\eta}_{a k l}=\bar{C}_{a b}^{c o n d} \bar{\eta}_{b i j}
$$

and describes the orientation of the condensate of instantons and antiinstantons.

Substituting Eqs.(8),(9) into Eq.(5) and using the identity [9]

$$
\eta_{a \mu \nu} \eta_{a i j}+\bar{\eta}_{a \mu \nu} \bar{\eta}_{a i j}=2\left(\delta_{i \mu} \delta_{j \nu}-\delta_{j \mu} \delta_{i \nu}\right)
$$

one finds that the Lagrangian (5) may be written as

$$
\Delta \mathcal{L}_{Y M}=-f h^{i \mu} h^{j \nu} F_{\mu \nu}^{i j} .
$$

Remember that the Latin letters $i, j$ label the indexes of variables in the isotopic space while the Greek letters $\mu, \nu$ label the indexes in the coordinate space. The symbols $\eta_{a i j}$ play a role of the generators of the gauge transformations, see Eq.(3), while the symbols $\eta_{a \mu \nu}$ describe the orientation of instantons in the coordinate space, see Eq.(住). Eq. (10) gives a match between the indexes of isotopic and coordinate spaces. It makes it useful to consider the matrix $h^{i \mu}$ in Eq.(11) with one Latin index and one Greek one. This matrix plays a role of the order parameter of the problem.

From Eq.(11) one deduces that there appears the corresponding term in the action:

$$
\Delta S=-f \int h^{i \mu} h^{j \nu} F_{\mu \nu}^{i j} \operatorname{det} h d^{4} x .
$$

This action is invariant under two types of transformations. First, it preserves gauge symmetry. Gauge transformations have a form

$$
F_{\mu \nu}^{\prime i j}=U^{i k}(x) U^{j l}(x) F_{\mu \nu}^{k l}, \quad h^{i \mu}=U^{k i}(x) h^{k \mu} .
$$

Second, it is invariant under the transformations of the coordinates $x^{\mu} \rightarrow x^{\prime \mu}$

$$
F_{\mu \nu}^{i j}=\frac{\partial x^{\rho}}{\partial x^{\prime \mu}} \frac{\partial x^{\sigma}}{\partial x^{\prime \nu}} F_{\rho \sigma}^{i j}, \quad h^{i \mu}=\frac{\partial x^{\prime \mu}}{\partial x^{\nu}} h^{i \nu} .
$$

The factor $\operatorname{det} h\left(\equiv \operatorname{det} h_{\mu}^{i}\right.$, where $h_{\mu}^{i}$ denotes the matrix inverse to $\left.h^{i \mu}: h^{i \mu} h_{\mu}^{j}=\delta_{i j}\right)$ in Eq.(12) compensates for the variation of the volume $d^{4} x$ due to the identity $\operatorname{det} h=\operatorname{det}\left[\partial x^{\mu} / \partial x^{\prime \nu}\right]$, which follows from Eq.(14).

Up to now we assumed the condensate to be homogeneous and the matrix $h^{i \mu}$ was considered as a global orthogonal matrix, $h^{i \mu} \in S O(4)$. This is true for some particular coordinate frame and particular gauge condition, see (13), (14). Similar consideration may be fulfilled for the case of the non-homogeneous condensate as well. In this case the order parameter may be shown to be described by the matrix $h^{i \mu}(x)$ as well, but there are two important distinctions. First, the matrix $h^{i \mu}(x)$ varies in space. For non-homogeneous condensate this variation is nontrivial, i.e. it can not be eliminated with the help of the transformations (13), (14). Second, $h^{i \mu}(x)$ is an arbitrary matrix having 16 independent parameters.

Consider the interaction of a weak and smooth gauge field with the non-homogeneous condensate which also varies smoothly in space. Then it may be shown that this interaction is described by the same action (12) assuming that $h^{i \mu}=h^{i \mu}(x)$ is a function of $x$. A simple argument in favour of this result is a fact that locally, at any point $x_{0}$, the order parameter $h^{i \mu}\left(x_{0}\right)$ may be transformed with the help of the coordinate transformation (14) to be an orthogonal matrix $h^{i \mu}\left(x_{0}\right) \in S O(4)$. Then the expression (11) for the Lagrangian is valid in the vicinity of $x_{0}$. The point $x_{0}$ is arbitrary, therefore we can integrate the Lagrangian evaluating the action (12).

The action (12) depends on the vector potential $A_{\mu}^{i j}(x)$ and the matrix $h^{i \mu}(x) . A_{\mu}^{i j}(x)$ is a slowly varying vector potential having the trivial topological structure (at least on the microscopic level). In contrast to that $h^{i \mu}(x)$ describes the orientation of polarized instantons and antiinstantons which are the degrees of freedom of the field with nontrivial topological structure. This allows us to consider $A_{\mu}^{i j}(x)$ and $h^{i \mu}(x)$ as separate variables. Note that for the considered weak field the term quadratic in 
$F_{\mu \nu}^{i j}$ given by the Lagrangian (2) is much smaller compared to the linear term (12). Therefore we can neglect the action coming from Lagrangian (2).

The weak and smooth nature of the field permits one to use the classical approximation:

$$
\begin{aligned}
& \delta(\Delta S) / \delta A_{\mu}^{i j}=0, \\
& \delta(\Delta S) / \delta h^{i \mu}=0 .
\end{aligned}
$$

Eq.(15) gives the relation between $h^{i \mu}$ and $A_{\mu}^{i j}$

$$
\nabla_{\mu}\left(\left(h^{i \mu} h^{j \nu}-h^{j \mu} h^{i \nu}\right) \operatorname{det} h\right)=0 .
$$

Here $\nabla_{\mu}$ is the covariant derivative in the gauge field $\left(\nabla_{\mu}\right)^{i j}=\delta_{i j} \partial_{\mu}+A_{\mu}^{i j}$. In order to present Eq.(17) in a more convenient form let us define three quantities, $g_{\mu \nu}, \Gamma_{\mu \nu}^{\lambda}$, and $R_{\rho \mu \nu}^{\lambda}$ :

$$
\begin{aligned}
g_{\mu \nu} & =h_{\mu}^{i} h_{\nu}^{i}, \\
\Gamma_{\mu \nu}^{\lambda} & =h^{i \lambda} h_{\mu}^{j} A_{\nu}^{i j}+h^{i \lambda} \partial_{\nu} h_{\mu}^{i}, \\
R_{\rho \mu \nu}^{\lambda} & =h^{i \lambda} h_{\rho}^{j} F_{\mu \nu}^{i j} .
\end{aligned}
$$

Remember that the space-time under consideration is basically flat and Eqs.(18), (19), (20) define the left-hand sides.

From (18), (19) we find that Eq.(17) may be presented in the form: $g_{\lambda \sigma} \Gamma_{\mu \nu}^{\lambda}=(1 / 2)\left(\partial_{\mu} g_{\sigma \nu}+\partial_{\nu} g_{\sigma \mu}-\partial_{\sigma} g_{\mu \nu}\right)$. demonstrating that we may consider $g_{\mu \nu}$ as a metric and $\Gamma_{\mu \nu}^{\lambda}$ as a Christoffel symbol. Moreover, one finds that the quantity $R_{\rho \mu \nu}^{\lambda}$ introduced in Eq.(20) turns out to be equal to the Riemann tensor: $R_{\rho \mu \nu}^{\lambda}=\partial_{\mu} \Gamma_{\rho \nu}^{\lambda}-\partial_{\nu} \Gamma_{\rho \mu}^{\lambda}+\Gamma_{\sigma \mu}^{\lambda} \Gamma_{\rho \nu}^{\sigma}-$ $\Gamma_{\sigma \nu}^{\lambda} \Gamma_{\rho \mu}^{\sigma}$. Considering now the second classical equation (16) one verifies with the help of Eqs.(18),(20) that it results in the Einstein equations of general relativity in the absence of matter: $R_{\mu \nu}-(1 / 2) g_{\mu \nu} R=0$.

We come to the important conclusion. If IAP takes place in the $\mathrm{SO}(\mathbb{4})$ gauge theory then the classical approximation for this gauge theory may be described via the variables of Riemann geometry for which the Einstein equations are valid. These equations imply in particular that there exist gravitational waves. That is a pleasant surprise since the initial gauge theory possesses no graviton on the basic level. Graviton appears due to excitation of the condensate of instantons and antiinstantons.

Consider the action (12) when the classical Eq. (15) is valid. It is clear from $(18),(\sqrt{19}),(20)$ that it is identical to the Lagrangian of general relativity (1). The gravitational constant turns out to be

$$
k^{-1}=16 \pi f .
$$

This relation shows that a radius and separation of instantons which give the contribution to the constant $f$, see Eqs.(5),(8), are comparable to the Plank radius.

We see how the distinctions (i), (ii), (iii) discussed at the beginning of the paper are eliminated by IAP. The major problem is (i), the compactness of the group of local transformations. IAP solves it in a peculiar manner. The variables of the gauge theory are expressed, see (18), (19), (20), via the variables of Riemann geometry. This geometry deals with the invariance with respect to transformations of a local reference frame. These transformations may be continued from Euclidean to Minkowsky space.
I thank my colleagues at A.F.Ioffe Institute for a stimulating scientific atmosphere. I thank V. V. Flambaum, C. J. Hamer, and O. P. Sushkov for helpful discussions. I appreciate the financial support from DITAC which made it possible to finish this work at School of Physics of UNSW, and the hospitality of their staff is acknowledged. The kind help of L.S.Kuchieva on all the stages of this work was vital.

(The paper is accepted for publication in Europhysics Letters, submitted in the final form 19 October, 1994.)

(a) On leave from: A. F. Ioffe Physical-Technical Institute of Russian Academy of Sciences, 194021 St.Petersburg, Russian Federation

[1] C. N. Yang, R. Mills, Phys. Rev. 96 (1954) 191.

[2] A. A. Belavin, A. M. Polyakov, A. S. Schwartz, and Yu.S.Tyupkin, Phys. Lett. 59B (1975) 85.

[3] L. D. Faddeev, A. A. Slavnov, Gauge fields: introduction to quantum theory (Benjamin/Cummings, London 1980).

[4] A. Ashtekar, Phys.Rev.Lett. 57 (1986) 2244.

[5] I. L. Buchbinder, S. D. Odintsov, and I. L. Shapiro, Effective action in quantum gravity, (IOP, Bristol, 1992).

[6] L. D. Landau, E. M. Lifshitz, The Classical Theory of Fields (Pergamon Press, Oxford and New York, 1971).

[7] C. Callan, K. Dashen, and D. Gross, Phys. Rev. D 17 (1978) 2717.

[8] A. I. Vainshtein, V. I. Zakharov, V. A. Novikov, and M. A. Shifman, Usp. Fiz. Nauk 136 (1982) 553.

[9] G. 't Hooft, Phys. Rev. D 14 (1976) 3432. 